\title{
Promoting healthy teenage behaviour across three European countries through the use of a novel smartphone technology platform, PEGASO fit for future: study protocol of a quasi-experimental, controlled, multi-Centre trial
}

\author{
Elisa Puigdomenech ${ }^{1,2^{*+}}$ D , Anne Martin ${ }^{3+}$, Alexandra Lang ${ }^{4}$, Fulvio Adorni ${ }^{5}$, Santiago Felipe Gomez ${ }^{6,7}$, \\ Brian McKinstry ${ }^{8}$, Federica Prinellii ${ }^{9}$, Laura Condon ${ }^{10}$, Rajeeb Rashid ${ }^{11}$, Maurizio Caon ${ }^{12}$, Sarah Atkinson ${ }^{13}$, \\ Claudio L. Lafortuna ${ }^{14}$, Valentina Ciociola ${ }^{15}$, Janet Hanley ${ }^{16}$, Lucy McCloughan ${ }^{17}$, Conxa Castell ${ }^{18}$, \\ Mireia Espallargues ${ }^{1,2}$ and on behalf of the PEGASO Fit For Future Consortium
}

\begin{abstract}
Background: Behaviour change interventions targeting physical activity, diet, sleep and sedentary behaviour of teenagers show promise when delivered through smartphones. However, to date there is no evidence of effectiveness of multicomponent smartphone-based interventions. Utilising a user-centred design approach, we developed a theory-based, multi-dimensional system, PEGASO Fit For Future (PEGASO F4F), which exploits sophisticated game mechanics involving smartphone applications, a smartphone game and activity sensors to motivate teenagers to take an active role in adopting and maintaining a healthy lifestyle. This paper describes the study protocol to assess the feasibility, usability and effectiveness (knowledge/awareness and behavioural change in lifestyle) of the PEGASO system.

Methods: We are conducting a quasi-experimental controlled cluster trial in 4 sites in Spain, Italy, and UK (England, Scotland) over 6 months. We plan to recruit 525, in a 2:1 basis, teenagers aged 13-16 years from secondary schools. The intervention group is provided with the PEGASO system whereas the comparison group continues their usual educational routine. Outcomes include feasibility, acceptance, and usability of the PEGASO system as well as between and within group changes in motivation, self-reported diet, physical activity, sedentary and sleeping behaviour, anthropometric measures and knowledge about a healthy lifestyle.
\end{abstract}

(Continued on next page)

\footnotetext{
* Correspondence: epuigdomenech@gencat.cat

${ }^{\dagger}$ Elisa Puigdomenech and Anne Martin contributed equally to this work ${ }^{1}$ Agency for Health Quality and Assessment of Catalonia (AQuAS), Catalan Department of Health, Roc Boronat 81-95, 2nd floor, 08005 Barcelona, Spain ${ }^{2}$ Health Services Research on Chronic Patients Network (REDISSEC), Instituto de Salud Carlos III, Roc Boronat 81-95, 2nd floor, 08005 Barcelona, Spain Full list of author information is available at the end of the article
} Commons Attribution 4.0 International License (http://creativecommons.org/licenses/by/4.0/), which permits unrestricted use, distribution, and reproduction in any medium, provided you give appropriate credit to the original author(s) and the source, provide a link to the Creative Commons license, and indicate if changes were made. The Creative Commons Public Domain Dedication waiver (http://creativecommons.org/publicdomain/zero/1.0/) applies to the data made available in this article, unless otherwise stated. 
(Continued from previous page)

Discussion: PEGASO F4F will provide evidence into the cross-cultural similarities and differences in the feasibility, acceptability and usability of a multi-dimensional smartphone based behaviour change intervention for teenagers. The study will explore facilitating factors, challenges and barriers of engaging teenagers to adapt and maintain a healthy lifestyle when using smartphone technology. Positive results from this ICT based multi component intervention may have significant implications both at clinical level, improving teenagers health and at public health level since it can present an influential tool against the development of chronic disease during adulthood.

Trial registration: https://clinicaltrials.gov Registration number: NCT02930148, registered 4 October 2016.

Keywords: Adolescents, Obesity prevention, Behaviour change, Physical activity, Diet, Sleep, Sedentary behaviour, Health promotion, Mobile health, mHealth, eHealth, Smartphone application, Serious game,

\section{Background}

Adolescence is a period characterised by significant changes at physical and psychological levels [1]. It is a life-course stage vulnerable to acquiring unhealthy behaviours such as insufficient levels of physical activity and excess of sedentary behaviour, poor diet and sleep deprivation [2]. Several studies highlight the importance of adopting and maintaining healthy lifestyles amongst young people to ensure physical health and mental wellbeing during adolescence and adulthood [3-6]. Interventions that facilitate healthy dietary habits [7]; regular physical activity [8]; adequate sleep duration [9], and reduced sedentary behaviour $[10,11]$ were shown to be effective in preventing and treating obesity and other risk factors of non-communicable conditions such as type II diabetes, cardiovascular disease or cancer. However, available evidence is still overall considered to be of low to moderate quality $[12,13]$.

In Europe the proportion of overweight individuals in the general population is a relevant public health problem. According to the World Health Organization (WHO), the prevalence of overweight (body mass index $>85$ th percentile) among European 13 -year-olds is $27 \%[6,14]$. Overweight during childhood and adolescence is a risk factor for obesity in adulthood [15] and thus overweight control by empowering healthy lifestyles habits among adolescents is generally considered a fruitful strategy of public health promotion. Several social, school-based and media campaigns have been conducted aiming to motivate behaviour change [12]. However, in children and adolescents, evidence of a reduction in overweight and obesity prevalence is lacking [16] and the prevalence of related unhealthy behaviour is still very high [17]. This suggests that innovative intervention strategies, more engaging and attractive for children and adolescents, are needed.

In Europe, as well as in other regions, the development of electronic and mobile Health (eHealth and mHealth) has largely focused on the management of chronic diseases [18-21]. The combination of these and further integrated health applications (apps) is referred to as Health 4.0, which is the ultimate goal of ubiquitous, intelligent and interactive health service provision [22]. Nowadays, adolescents live in a highly technological world rapidly adopting information and communication technologies (ICTs) in general, and especially mobile devices such as smartphones, tablet computers [23] and electronic bracelets [24]. Digital platforms allow adolescents to go beyond face-to-face interaction, to be connected online and to have access to information from social media and networks, independently from the socioeconomic or familiar strata [25]. In this context, public health authorities have an opportunity to disseminate key messages to adolescents to promote healthy decision-making and lifestyles in a creative and innovative way relevant to the intended user population [26].

Recent reviews show that besides being feasible and acceptable, the use of mobile and wireless technologies in adolescents can help to prevent and treat overweight condition and obesity among children and adolescents [27-29]. The literature review undertaken by Dute and collaborators on mHealth based interventions to promote healthy habits among adolescents, showed that 'only 4 apps were developed specifically for adolescents. All apps were tested on a small scale and for a short period' [29]. Mobile apps for promoting healthy eating and physical activity tend to provide the user with the opportunity to set personal goals, to self-monitor their healthy behaviour and to receive tailored feedback. Although known to be effective, other behaviour change techniques such as social support are rarely present in health promotion apps for adolescents to date [29].

This paper presents the study protocol of a crosscultural mHealth behaviour change intervention for use by teenagers, PEGASO Fit For Future (PEGASO F4F), designed to provide proactive health promotion in a real-world environment for overweight and obesity prevention. The study aims to understand and test the acceptance of a suite of integrated technologies (PEGASO system), the user experience and the effects of the PEGASO system on: 
a) change of knowledge about healthy eating, physical activity, sitting (sedentary behaviour) and sleeping habits

b) motivation to change obesity-related lifestyle behaviours

c) behavioural change in obesity-related lifestyle behaviours

\section{Methods}

\section{Design and settings}

A quasi-experimental cluster controlled trial was undertaken to evaluate an ICT-based intervention over 6 months, i.e., the PEGASO System, to promote healthy habits among adolescents. This study includes adolescents of 12 schools from four sites: England (UK), Scotland (UK), Lombardy (Italy) and Catalonia (Spain).

The target sample size of 525 with 2:1 allocation (intervention: control) was designed to be large enough in each school to be reasonably sure that large scale recruitment was possible, to have a representation from different socioeconomic groups, to allow for attrition and to give some indication of variance in the various proposed outcome measures which would permit a sample size calculation for a future cluster randomized controlled trial.

Ethical approval was granted by the clinical research ethics committee (CEIC) of all four intervention sites: South East Scotland Research Ethics Committee in the UK, Istituto di Ricovero e Cura a Carattere Scientifico (IRCCS) Policlinico of Milan in Italy and CEIC of Institut d'Investigació en Atenció Primaria de Salut Jordi Gol (IDIAP Jordi Gol) in Spain.

\section{Recruitment and group allocation}

Potential participants were identified in schools, which were approached through researcher's contacts. The selection of schools was determined by our aim to include students from a range of socioeconomic backgrounds. Different recruitment strategies were applied in the schools. In two sites, whole classes were recruited to the intervention group and whole classes to the control group (from the same school or from a different one). Another recruitment strategy was that the intervention was offered to all students in eligible year groups and volunteers were sought with the first responders being chosen. Participants for the control group were similarly chosen from another school. Participant recruitment took place from September to November 2016.

\section{Participant selection criteria}

Eligible for inclusion in this study were teenagers aged 13 to 16 years (inclusive) without physical or psychological conditions that would prevent the understanding of and their participation in the intervention, who were able to participate in the study for 6 months and had an adequate proficiency in the local language (Italian, Catalan/Spanish and English). Although it is generally accepted that adolescence extends beyond this age, range 13-16 years was chosen as the most feasible age range to introduce this type of intervention in a school context.

\section{Intervention}

The PEGASO system is based on the comprehensive Behaviour Change Wheel (BCW) framework and the Capability-Opportunity-Motivation-Behaviour (COM-B) system [30]. This provides the theoretical grounding for the content and implementation approach of the behaviour change interventions embedded within the components of the PEGASO system (Table 1).

The innovative key component of the PEGASO system is the behaviour recognition system, which allows detection and evaluation of participants' real-time behaviour (collected through other components of the PEGASO system). A summary of the obesity-related behaviours targeted by the PEGASO system is shown in Table 2.

Participants have the opportunity to choose one target behaviour at the time. When selecting a new target behaviour, participants are asked to complete the COM-B questionnaire [30]. The answers for each question subset guide which component of the COM-B behaviour system is adopted to target the content of the behaviour change intervention. For instance, the messaging framework in the PEGASO Companion App selects messages that are associated with participants' preferences expressed in the COM-B questionnaire. This provides an intervention that is personalised to the different needs of the individual intervention participant.

The PEGASO system was developed using a usercentred design approach aiming to deliver a product and interactions which are attractive to adolescents and which enabled them to connect with their peers.

\section{Components of the PEGASO platform}

The PEGASO system includes physical activity monitoring devices, a web portal, and up to six apps: Companion app, Challenges app, EDiary app, Dashboard app, serious game and Report app.

\section{Physical activity monitors}

The PEGASO system has two sets of physical activity monitors; a smart garment and a wrist-worn activity tracker (Fig. 1). Participants are asked to wear the smart garments as often as possible in typical environments when being physically active e.g. at school during physical education, after school sports club, outdoors, commuting to school, while exercising, at home and sleeping. The smart garment is a T-shirt/cropped top with embedded textile electrodes and a monitoring 
Table 1 Behaviour change technique (BCT) within each PEGASO system component

\begin{tabular}{|c|c|}
\hline Behaviour change technique & Operationalisation in the PEGASO system \\
\hline \multicolumn{2}{|l|}{ Companion App } \\
\hline $\begin{array}{l}\text { Information about health consequences, Prompt/Cues, Instruction on how } \\
\text { to perform a behaviour }\end{array}$ & News stream UI \\
\hline Feedback on behaviour & Energy bar \\
\hline Self-monitoring of behaviour & Interaction with specific UI elements in the News Stream \\
\hline Social support & Friends UI \\
\hline Problem solving & Messages and reminders \\
\hline \multicolumn{2}{|l|}{ EDiary App } \\
\hline Self-monitoring of behaviour, Feedback on behaviour & Diversity and Balance index and Food groups consumption \\
\hline Prompt/Cues & Current target behaviour \\
\hline Credible source & Companion Avatar \\
\hline Instruction on how to perform a behaviour & Companion Avatar suggestions \\
\hline \multicolumn{2}{|l|}{ Challenges App } \\
\hline Feedback on behaviour & Feedback on the performance in the challenge \\
\hline Prompt/Cues & New challenge available \\
\hline Self-monitoring of behaviour & Challenge status \\
\hline Instruction on how to perform a behaviour & Challenge description \\
\hline Social support & Competitive and collaborative challenges \\
\hline Goal setting (behaviour) & Selection of challenge \\
\hline Goal setting (outcome) & Selection of challenge \\
\hline \multicolumn{2}{|l|}{ Dashboard App } \\
\hline Self-monitoring of behaviour, Feedback on behaviour & Presentation of performances in activities related to physical activity \\
\hline \multicolumn{2}{|l|}{ Serious Game } \\
\hline Information about nutrients in food & $\begin{array}{l}\text { Mini-games (Scavenge mini-game, Research mini-game, NPC mini- } \\
\text { game, Laptop mini-game) }\end{array}$ \\
\hline Feedback on behaviour & Energy bar, score and points, mini-games, game results \\
\hline Feedback on the outcomes of the behaviour & Energy bar metaphor \\
\hline Credible source & Game narrative \\
\hline Adding objects to the environment & Game narrative \\
\hline \multicolumn{2}{|l|}{ PEGASO Web Portal } \\
\hline Information about health consequences & Blog information and training modules \\
\hline Self-monitoring of behaviour Feedback on behaviour & ZivaCare module \\
\hline Feedback on the outcomes of the behaviour & Blog information contact with PEGASO experts and training modules \\
\hline Credible source & Contact with experts \\
\hline Instruction on how to perform a behaviour & Blog information and training modules \\
\hline Social support & Contact with peers, tutors and expert \\
\hline \multicolumn{2}{|l|}{ Report App } \\
\hline Self-monitoring of behaviour Feedback on behaviour & Display of stats about user behaviours \\
\hline Social support & Provision of the possibility to interact with the family doctor \\
\hline
\end{tabular}

UI: User interaction

device (data logger). The data logger attaches onto the front side of the T-shirt by using standard snap buttons. The smart garments measure heart rate, physical activity levels, and postures [31]. The activity bracelet monitors physical activities and sleep and participants are asked to wear the device daily during day and night, while removing it for swimming, showering and bathing. Data collected through the smart garment and bracelet is transferred to the smart phone and linked to the Companion App, Dashboard App and the serious game. 
Table 2 Target behaviours of the PEGASO system and behavioural goals

\begin{tabular}{ll}
\hline Target behaviour & Behavioural goals \\
\hline Dietary target behaviours & - Fruit consumption of $\geq 2$ servings $(250-375 \mathrm{~g}) /$ day \\
& - Vegetable consumption of $\geq 2$ servings ( $300-450 \mathrm{~g}) /$ day \\
& - Reduced intake of sugar-sweetened beverages \\
& - Daily breakfast consumption \\
& - Reduced intake of fast food \\
& - Reduced consumption of sweet and salty high-energy snack high-energy \\
& - 60 min of daily physical activity of moderate-to-vigorous intensity (i.e. involving energy expenditure over 4 METs) \\
& - Undertake 12.000 steps/day \\
Physical activity target behaviours & - Daily active transport to and from school \\
& - less than $45 \%$ of after-school time daily spent in sedentary activities ( $\leq 1.5$ METs) \\
Sedentary behaviour & -Daily sleep duration of at least $8 \mathrm{~h}$ \\
Sleep behaviour &
\end{tabular}

METs Metabolic Equivalents

\section{Web portal}

The web portal allows participants to socially interact with each other and to access tutorials on how to improve their diet and increase their physical activity levels through educational training modules. The web portal is accessible from both mobile devices and computers.

\section{Companion app}

The objective of the Companion app is to encompass all other PEGASO apps (Fig. 2). The Companion App aims to act as a personal digital friend which coaches, cares, and empowers participants in developing healthy habits. It aims to provide the participant with a dynamic stream of educational and motivational messages and updates about their achievements and their friends' shared achievements. Data acquired via physical activity monitors is presented to the participant via a specific interface presented within the Companion app. The Companion app also includes a reward system and the gamification module to allow the participants to: a) achieve one time rewards (e.g., FitCoins, badges, experience points) for the selected target behaviour and b) achieve cyclic events.

\section{Challenges app}

Through the Challenges app participants can create and accept different types of challenges: individual, competitive or collaborative challenges related to the target behaviours (Table 2). Participants are asked to engage in healthy eating and/or physical activity behaviour to meet the selected challenge. Participants gain points when a challenge is successfully completed (e.g. engaging in 30 min of moderate to vigorous physical activity).

\section{EDiary app}

This app allows the participant to enter information about their diet and provides feedback about the balance and diversity of consumed food (Fig. 3). It aims to

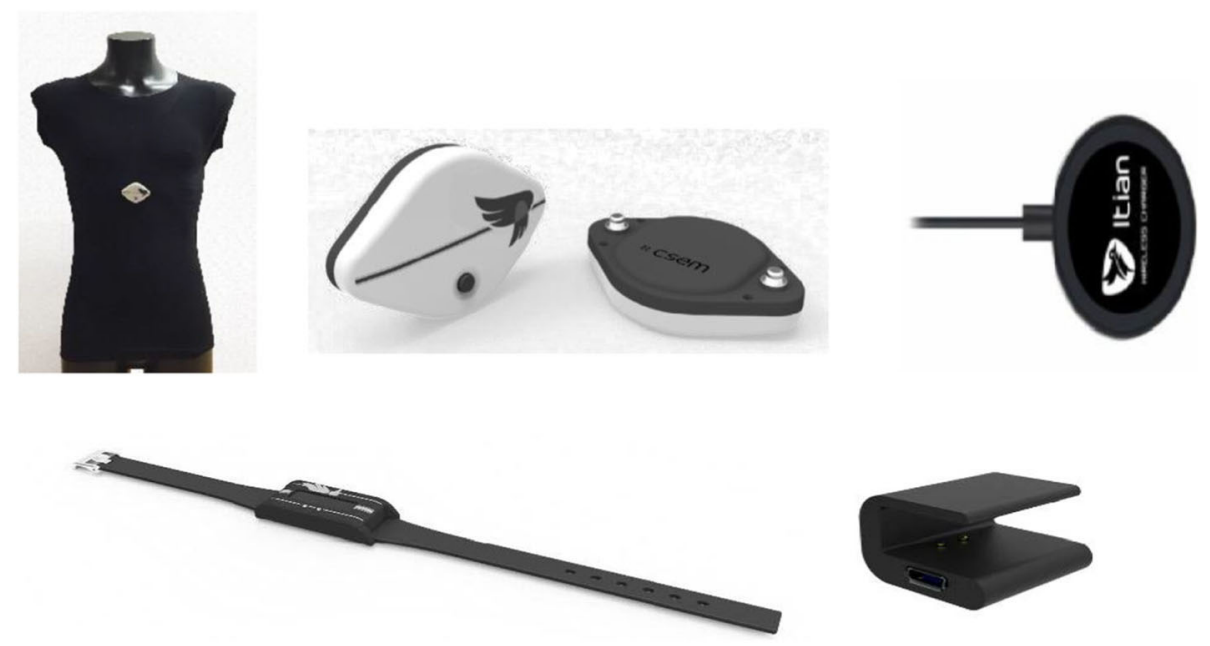

Fig. 1 This figure shows PEGASO smart garment and a wrist-worn activity tracker 

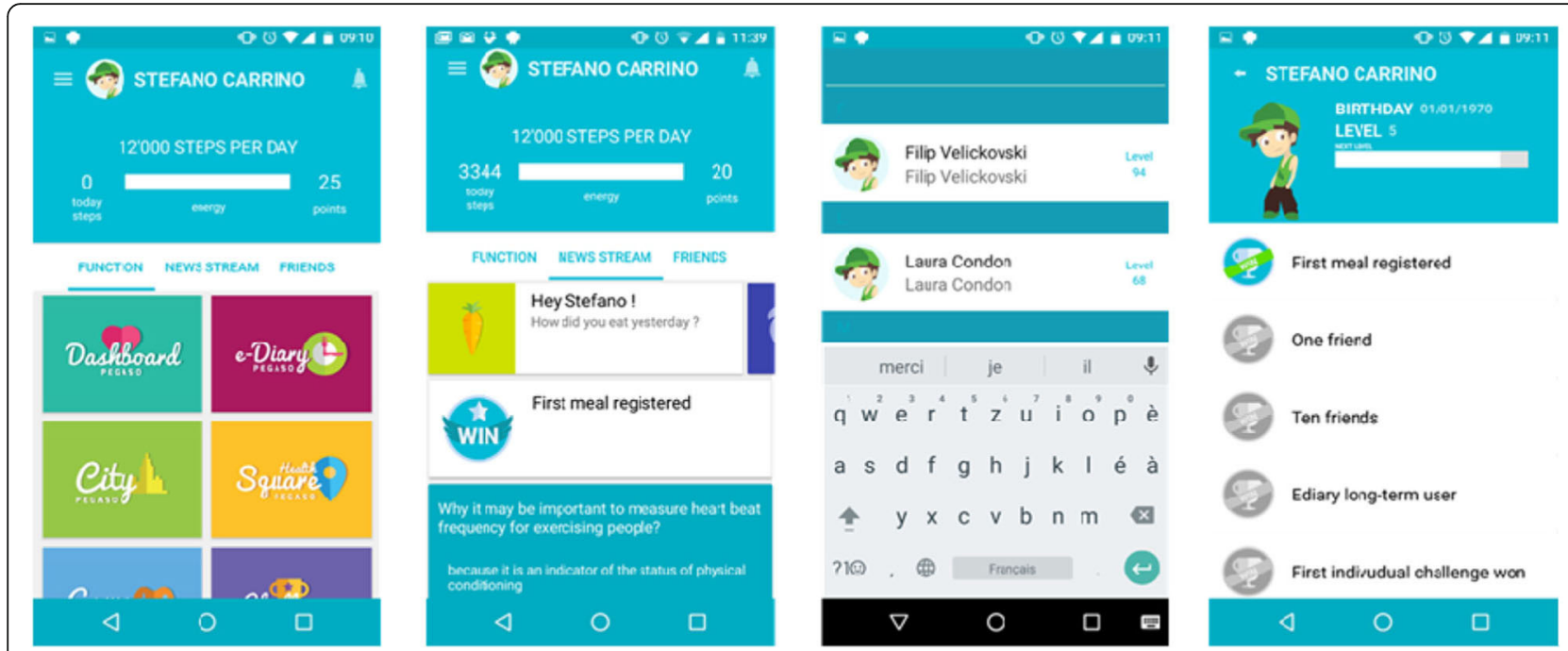

Fig. 2 This figure shows some screen shots of the Companion application

provide guidance on recommended food groups for the next meal based on participants' behaviour. The EDiary app is gamified aiming to improve the participants' experience through introducing an element of fun that can sustain engagement within the app and daily eating habits.

\section{Dashboard app}

This app allows participants to self-monitor their sleep time and physical activity behaviours: number of steps and minutes spent in moderate-to-vigorous physical activity (MVPA), type of activities made during the course of the day. Activities requiring energy expenditure above 4 METs (as estimated from outputs of the sensor devices) were considered as MVPA, which allows for the higher resting metabolic rate of adolescents [32].

\section{Serious game}

The serious game aims to serve a central role as the motivational component of PEGASO. As such, it needs to entertain and engage the player, whilst utilising the PEGASO system to capture information about lifestyles and provide relevant feedback to encourage positive health behaviour changes. There are two central behavioural mechanisms within the game: 1) An "energy bar", used up by the player's actions in the game, and replenished when achieving their behavioural goals such as increased physical activity or improved diet, and 2) the
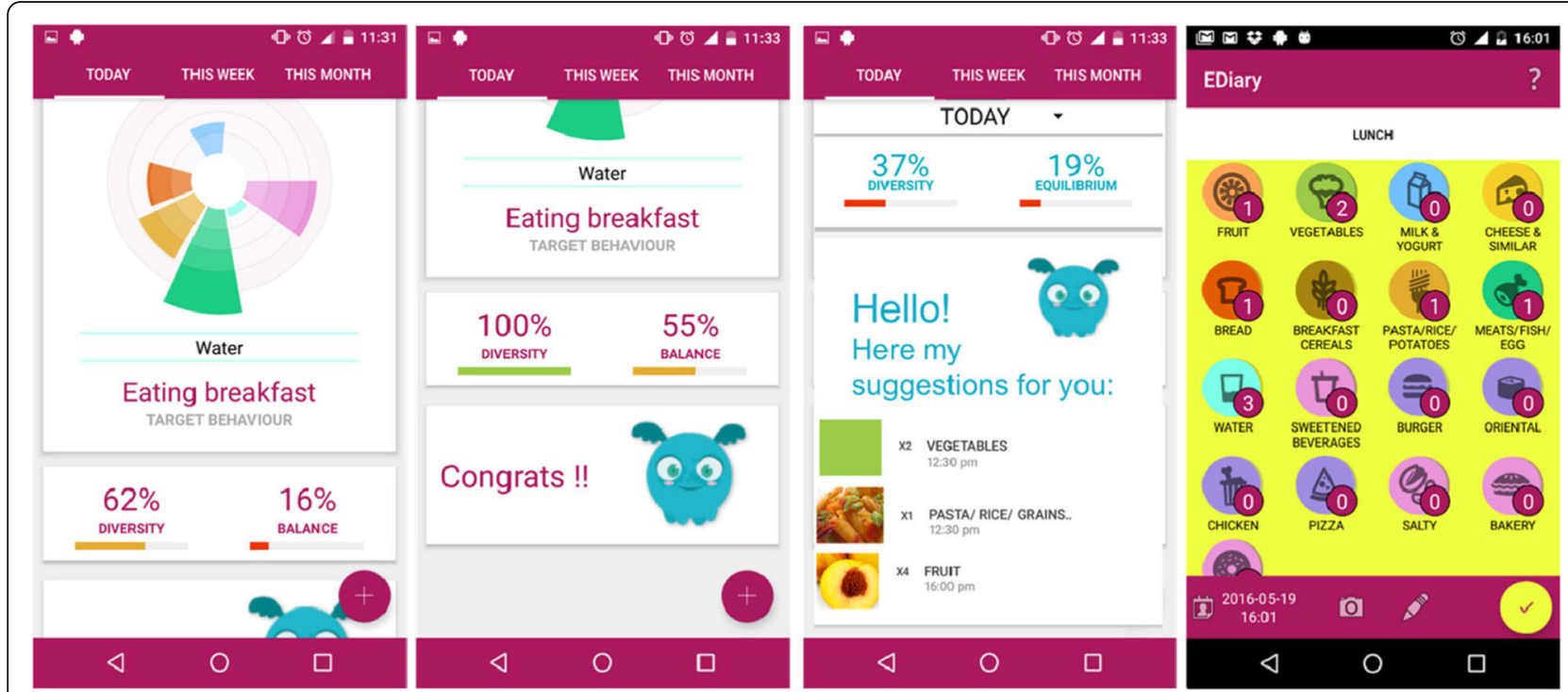

Fig. 3 This figure shows some screen shot of the EDiary application 
game implements "research" mechanics that require the player to apply and develop their nutritional knowledge of various food sources.

\section{Report app}

Although not being tested in the present study, PEGASO consortium developed this app, which aims to connect the PEGASO system with the healthcare system in counties with electronic health records supporting user data sharing (in this study Italy and Spain). Through a bridge system within the Report app, it is planned that data collected by the PEGASO system will be extracted and shared with the health professional in the form of a summary report which will be added to the personal health folder.

\section{Procedures}

Eligible participants are introduced to the PEGASO system after they and their parents/guardian provided written and signed consent, and after baseline measurements are collected. Participants are provided with a smart phone (running as OS Android, version from 4.4 to 6.0) with the PEGASO apps installed for the duration of the study.

Given the complexity of the PEGASO system, the components are introduced in two sessions within the same first week. The physical activity monitors are introduced to the participants at the mid-point assessment time at month 4 of the study. This time point was chosen to reduce the burden on the schools and participants, allocating time and resources in addition to the baseline measures, mid-point and follow-up assessment contact points, whilst additionally avoiding conflict with exam timetables and extended holiday periods (e.g. summer break). In addition to familiarising the participants with the functionalities and use of the garment and activity monitors, researchers assist participants in connecting the activity monitors with individual smart phones. All introduction sessions across the four study sites provide a mixture of interactive workshops, educational games, and replicate the method as much as possible within the constraints of the school requirements in different countries.

Participants are offered support during the study to facilitate understanding of how to use the PEGASO system and to trouble-shoot any issues arising. Participants are invited to join a site specific Google Hangout group to allow timely communication with the field researchers who provide solutions to technical issues whenever possible. Teachers at schools are offered a brief training session to be able to answer simple questions related to the use of the PEGASO system. They are asked to direct the participants to the web portal and the FAQ link for questions they cannot answer.
The role of teachers is to support students over the testing period, and to facilitate the communication flow among field researchers, parents and teenagers. During on-site contact points an interaction with teachers is expected; they will have the opportunity to indicate to the field researchers any comments, doubts or conflicts including maladaptive behaviours.

\section{Comparison group}

Schools and participants of the comparison group are given information about the purpose of the study; excluding details about the PEGASO system, at a single face-to-face session held by the field researchers. The comparison schools and participants are asked to continue their routine daily physical and educational activities related to leading a healthy lifestyle.

\section{Data collection and security}

Data are directly recorded by the platform or on-line questionnaires and stored in the Amazon Cloud. The confidentiality of collected data is ensured in accordance with the provision of European current legislation on personal data protection: 95/46/EC EU Directive (DPD) on Data Protection [33] and the EU 2016/680 General Data Protection Regulation [34].

\section{Outcomes and variables}

The first block of outcomes that will be assessed are related to recruitment and adherence to the intervention, namely recruitment rate, study retention, adherence to the intervention and attitudes to the different components of the intervention.

We aim to test a range of outcomes related to individual lifestyle (dietary habits, physical activity, sedentary behaviour, and sleeping habits), knowledge/awareness about these health habits and user experience of the platform. Individual lifestyle and knowledge/awareness about health habits are assessed at baseline and at the end ( 6 months) of the intervention both in the comparison and intervention group. User experience is assessed at month 2, 4 and 6 only in the intervention group. Outcomes, instruments and assessment points during the study are detailed in Table 3.

Individual lifestyle outcomes are measured through on-line questionnaires. Dietary habits are assessed through the KIDMED, a 16 item semi-quantitative food habits questionnaire which aims to assess dietary patterns among children, adolescents and young adults [35]. Additional questions are also included to assess the consumption of fast food and sugar-sweetened beverages, breakfast skipping and snacking behaviour. Physical activity is assessed through the Physical Activity Questionnaire for Adolescents (PAQ-A) [36]. Additional questions and an extra question from PAQ-elementary 
Table 3 Outcomes, instruments and assessment points in the PEGASO study

\begin{tabular}{|c|c|c|c|c|c|}
\hline Outcome & Instrument & Asse & essm & ent pc & \\
\hline & & BL & M2 & M4 & M6 \\
\hline Dietary habits & $\begin{array}{l}\text { Mediterranean Diet Quality Index in children and adolescents (KIDMED questionnaire) + } 5 \text { additional } \\
\text { questions }\end{array}$ & $x$ & & & $x$ \\
\hline Physical activity & Physical Activity Questionnaire for Adolescents $(\mathrm{PAQ}-\mathrm{A})+3$ additional questionnaires & $x$ & & & $x$ \\
\hline Sedentary behaviour & Screen time-based sedentary behavior questionnaire (SSBQ) & $x$ & & & $x$ \\
\hline Sleeping habits & Sleep Habits Survey (SHS) & $x$ & & & $x$ \\
\hline Knowledge questionnaire & Ad hoc questionnaire & $x$ & & & $x$ \\
\hline Anthropometric Measurements & & $x$ & & & $x$ \\
\hline Height & Stadiometer SECA 213 & $x$ & & & $x$ \\
\hline WC & Flexible graduated measuring tape (SECA 201) & $x$ & & & $x$ \\
\hline Weight & England, Catalonia, Italy: Homologated & $x$ & & & $x$ \\
\hline $\mathrm{BMI}$ & electronic scale Tanita SC240MA & & & & \\
\hline$\%$ of body fat & Scotland: Tanita MC780MA Multi & $x$ & & & $x$ \\
\hline$\%$ of body water & Frequency Segmental Body Composition Analyser & $x$ & & & $x$ \\
\hline $\begin{array}{l}\text { Family affluence and self-reported health } \\
\text { status }\end{array}$ & Family Affluence Scale (FAS) + 1 question from the Social Functioning subscale -12 (SF-12) & $x$ & & & \\
\hline Motivation & Perceived Competence Scale & $x$ & $x$ & $x$ & $x$ \\
\hline Usability & System Usability Scale & & & $x$ & $x$ \\
\hline Emotional Response to the Platform & Emotional Metric Outcome questionnaire & & & $x$ & $x$ \\
\hline Satisfaction, Trust, Reputation & Ad hoc questionnaire & & & $x$ & $x$ \\
\hline User Experience & Focus Groups & & & $x$ & $x$ \\
\hline
\end{tabular}

In italics, outcomes assessd only in the intervention group. All the other outcomes were assessed in both, intervention and comparative group

Additional questions: Research team included additional questions in order to better assess the target outcome in case no validated questionnaire were found

Parent's undertook an ad-hoc questionanire in order to assess sociodemographic characteristics, Socio Economic Status (SES) and Family structure and living

conditions of the family

WC Waist Circumference, BMI Body Mass Index

BL Baseline, M2 Month 2, M4 Month 4, M6 Month6

are included to assess those issues related to PA not covered by the PAQ-A such as transportation to school. Sedentary behaviour is assessed by the screen timebased sedentary behaviour questionnaire (SSBQ) from the Healthy Lifestyle in Europe by Nutrition in Adolescence study (HELENA study), which has been validated among participants in the HELENA study against the use of an accelerometer. Participants are asked to report their habitual time devoted to several sedentary behaviours, mostly related to screen time: (a) TV viewing, (b) computer games, (c) console (video) games, (d) Internet for non-study reasons (hobbies), (e) Internet for study reasons and (f) study time (out of scholar schedule) [37]. Sleeping habits are assessed through four questions about sleep duration from the Sleep Habits Survey for Adolescents (SHSA), a validated self-reported survey that estimates sleep patterns in adolescents [38].

Knowledge/awareness about health habits are assessed through an ad-hoc questionnaire (quiz format) that covers knowledge of dietary habits, physical activity and sleeping behaviour. Most of the questions are from a questionnaire, which is validated in Italy [39] and some others are specifically created.

Motivation to maintain or increase healthy dietary habits and physical activity will be assessed through the
"Perceived Competence Scale" a short 4-item scale that assesses the degree to which participants feel confident about being able to make (or maintain) a change toward a healthy behaviour [40]. Adolescents' age, gender, perceived health status level and family affluence will be included in the on-line questionnaires. Parent's (or legal guardian's) educational level, occupation and familiar structure will be also documented only at baseline.

User experience (UX) refers to a person's total experience using a particular product, system or service [41]. The factors of UX to be investigated during the study include: emotion, engagement, trust, reputation, pleasure/ hedonism, usability, acceptability, accessibility, connectedness, context and target behaviours adherence. The PEGASO study aims to holistically assess how the teenagers experience the system in their everyday lives and where and how this experience facilitates use and where it can be improved. Mixed methods (quantitative and qualitative methodology) will be used to assess UX, specifically through self-reported questionnaires and focus groups or semi-structured interviews, respectively. The System Usability Scale (SUS) is a well established 10 item questionnaire with five response options to assess usability of a variety of products and services, where usability is a distinct concept within the overall 
understanding of UX [42]. SUS will be applied at month 4 to assess usability of the Companion and the game and at month 6 for the whole system. The Emotional Metric Outcome (EMO) questionnaire is a 16 item questionnaire to assess the emotional outcomes of interaction, both positive and negative [43]. The EMO will be undertaken at month 4 and 6 . A questionnaire has been created ad hoc in order to measure the satisfaction, trust and reputation of the platform. This comprises a 28 item questionnaire with 5 answer categories and is undertaken at months 4 and 6 .

\section{Focus groups}

Key contributors to understanding the UX of the PEGASO system such as; usability, acceptability, accessibility, context of use, connectedness, desirability and pleasure will also be assessed through focus groups; moreover they will be used to gain insight on users understanding of the system and their experience and motivation to engage/disengage with the PEGASO system and its different component parts (apps, games and sensors). Participants in the focus groups will be recruited from participants in the intervention group. A total of four focus groups per participating site (England, Lombardy and Catalonia) will be undertaken at month 4 and 6. Participants in focus groups at month 4 will have only tested the apps and the game of Pegaso, whilst participants on the Month 6 focus groups additionally will have used the sensors for 2 months. Each focus group will consist of 4-8 participants. The groups, whenever possible, will be gender-balanced with different levels of engagement to the PEGASO system. A trained moderator will guide the focus group following a script and will be assisted by an observer. All of them will be audio and/or videotaped and transcribed systematically, literally, and anonymously. An analysis will be made of thematic content, coding the data and grouping them into predefined categories based on topics.

\section{Anthropometric variables}

Anthropometric variables are assessed at baseline and at the end of the intervention as secondary outcomes. Body weight and percentage of body fat is assessed by bioelectric impedance analysis (BIA) performed using a homologated electronic scale (Tanita sc-240 ma) with the participant wearing light clothing and no shoes. Height is also measured with a portable system with the participant shoeless in the standing position (SECA 213). Body mass index (BMI) is calculated as weight $(\mathrm{kg})$ divided by height squared (m2). Waist circumference is measured using a flexible graduated measuring tape (SECA 201) with the patient in the standing position without clothing covering the midsection of the torso. All the anthropometric measurements are undertaken in the most private way possible.

\section{Data collected by the PEGASO system}

The interaction between the user and the Pegaso system is recognized through of a set of automatic engagement indicators measuring the actual usage of the system. For each participant, actions like interacting with the Companion app or registering a meal in the EDiary app are tracked and daily stored. The EDiary app records information about the reported meals composition, food groups and servings. The smartphone collects information about physical activity such as the number of steps or the total time spent doing physical activity. Further information on physical activity is collected from the 4th month by the introduction of a smart bracelet and smart sensor. General information about healthy habits is gathered through the "Self-Report Habit Index (SRHI)" adapted to include items that reflect self-identity [44]. The questionnaire appears as a notification inside the Companion app in between the achievement of a target behaviour and the selection of a new one. Self-awareness is recorded by the PEGASO system using the COM-B-Q Self-Assessment Questionnaire (Capability, Opportunity and Motivation for Behaviour) [45]. It is a questionnaire, based on the principles of behaviour change, about current behaviours and insight into aspects that need to be acted upon in order to achieve a pre-specified behavioural goal. The questionnaire appears as a notification inside the Companion app just after the selection of a new target behaviour.

\section{Analysis plan and statistical analysis}

The analysis plan will include a description of all data at every assessment point and an overall comparison of baseline characteristics between intervention group (IG) and comparison group (CG), as well as by sites and gender. Knowledge/awareness, lifestyles and anthropometric measurements will be compared at pre and post intervention (month 6) utilising an intention-to-treat (ITT) approach, independently from drop-outs and nonadherence. Relevant changes among lifestyles and knowledge will be defined according to current recommendations from international guidelines [46]. The effect of the intervention could be modified by gender, cultural and socioeconomic level, body mass index (BMI), as well as by the baseline lifestyles, which will be controlled in the analysis. Changes in motivation will be assessed in the four assessment points and compared to baseline. For the intervention group, the results from SUS, EMO, satisfaction, trust and reputation questionnaires will be compared from month 4 to month 6 . The level of engagement in the PEGASO platform will be assessed from indicators of usage at individual level, such as the 
number of days of interactions with the APPs or the daily number of steps, stored in the cloud.

Results will be reported as mean \pm standard deviation (or median, percentile 25 and 75) for quantitative variables or frequency and percentage distribution for qualitative variables. Quantitative variables will be tested for normality, log-transformed if needed, and accordingly analysed with parametric or non-parametric methods. Chi-squared test will be used to analyse the association between independent qualitative variables, along with the McNemar test for paired samples. The Student t-test will be used for the comparison of means between two groups, and the paired t-test will be applied to assess changes within one same group. Alternatively, the corresponding nonparametric tests will be used, as required. The relationship between quantitative variables will be analysed using Pearson's correlation coefficient or Spearman coefficient in the case of asymmetrically distributed variables. Techniques of principal component analysis (PCA) will be applied to identify patterns of variables best explaining outcomes of interest.

Multivariate linear regression analysis and logistic regression analysis will be used to analyse the variables determining the changes in physical activity and eating and sleeping habits. In order to analyse the effect of the intervention, comparison will be made of the changes observed in the CG versus the IG, with estimation of the Cohen $\mathrm{d}$ statistic, adjusting for the variables that may influence the results.

The level of statistical significance will be set at $\alpha=$ 0.05 and all tests will be two-tailed. Statistical analyses will be carried out using the software packages SPSS, version 22.0 (SPSS Inc., Chicago, IL) and Stata (StataCorp. 2009. Stata Statistical Software: Release 11. College Station, TX: StataCorp LP). Focus groups will analysis will be done with the support of Atlas. Ti /NVIVO and by triangulation of analyst.

\section{Discussion}

In general, and in the field of health and medicine in particular, in recent years information and communication technologies have experienced rapid growth. Some authors point out that the use of the internet and associated technologies such as computer apps represent a revolution within the medical field, since they are fast, versatile, manageable, illustrative tools that allow people to be empowered with their health [47]. However, this vast development of health apps and those related with healthy lifestyles promotion are not encompassed by clear and sufficient scientific evidence on health improvement. For instance, two recent systematic reviews concluded that mobile phone interventions can diminish sleep disorders and to enhance sleep quality [48] and mobile phone app-based interventions may be useful tools for weight loss [49]. However, some other reviews showed modest evidence on the efficacy of intervention based on health apps on the promotion of healthy habits related with diet, physical activity and sedentary behaviour in children and adults [50] or in improving preventive behaviours among adolescents [51, 52]. Schoeppe and collaborators concluded in their systematic reviews that multi-component interventions appear to be more effective than stand-alone app interventions [50]. In this sense, the PEGASO system could be considered as multi-component intervention, which includes several apps, a game and smart sensors, and which targets multiple health behaviours All the reviews highlighted the necessity to a) involve end-users on the development of app-based interventions and b) evaluate the health effects of app-based interventions by conducting experimental studies to obtain sufficient scientific evidence [48-51].

The first part of the PEGASO F4F study developed a multi-dimensional ICT based intervention that includes the use of smartphone applications, a game and the use of wearables to promote healthy habits among adolescents. An iterative pre- trial study has already been undertaken to achieve the user centred design approach of PEGASO F4F. The aim of this was to increase the likelihood that the system will meet the requirements of teenagers and be accepted by this specific group of end users. Volunteers from the 4 sites tested the platform in different moments of the development in an iterative process. Their feedback was used to assess user interaction and experience, usability and the security and confidentiality requirements of the PEGASO system.

The present study aims to test the methodology to investigate the effectiveness that the use of the Pegaso system will have on teenage health awareness and the improvement of healthy habits with regards to adherence to recommendations and habit formation in terms of dietary behaviour, physical activity and sedentary and sleeping behaviour among adolescents.

Moreover, the present study will assess the user experience through validated questionnaires and focus groups in order to receive feedback from end users when they are introduced to the new technology, over a shortterm period of use and then also over a longer period of time, 6 months after set up. The purpose of this is to understand how teenagers utilize proactive mHealth systems over time, understand the relationship between UX and health behaviours/ habit formation, and provide points of learning for industry looking to develop health ICT's and other interventions for young people; specifically adolescents.

The study follows most of the recommendations of the CONSORT for quasi experimental studies [53]; however, participants are not be blinded to the intervention 
due to the nature of the intervention. Self-reported data is used to measure main results related to healthy habits modification and knowledge. These are assessed through the validated questionnaires selected for this trial.

According to the availability of each site, two approaches for the selection of comparative groups were foreseen; the comparative group either came from the same school or from a different school. Both, clustered and non-clustered quasi-experimental studies have their own advantages and disadvantages. A possible crossover effects regarding the fact that both intervention and comparative groups are from the same schools may occur; social peer influence can be strongly associated to lifestyles and influence the results of the comparative group. However, we believe that adolescents tend to mainly interrelate with mates from their same class. Moreover, PEGASO F4F is not a school-based intervention itself; on the contrary, it was conceived as an individual intervention with several social components. By having both approaches it can provide us with some insight regarding the role of the social component and the impact if may have on the intervention.

The results of this study target improvements on dietary habits, physical activity and sedentary time and sleep quality among adolescents. Specifically results could lead to:

a) improvement in adolescent lifestyles (in terms of awareness, adherence and habit formation)

b) improved knowledge among adolescents about health and wellbeing and the impact of their personal day to day decisions on their health

c) an appreciation of how new technologies based on mHealth can assist them in their lifestyle choices and promote positive health behaviours.

d) Improved teenage mHealth interventions that are more appropriately designed to meet the specific needs of these young users

Positive results from this ICT based multi component intervention may represent an opportunity and influential tool against the development of chronic disease during adulthood.

\begin{abstract}
Abbreviations
App: Informatics application; BMI: Body Mass Index; EMO: Emotional Metric Outcomes; ICT: Information and Communication Technologies; mHealth: Mobile Health; PAQ-A: Physical Activity Questionnaire for Adolescents; PEGASO: Personalised Guidance Services for Optimising lifestyle in teen-agers through awareness, motivation and engagement; SHS: Sleep Habits Survey; SSBQ: Screen time-based sedentary behaviour questionnaire; SUS: System Usability Scale; UX: User Experience
\end{abstract}

\section{Acknowledgements}

The authors gratefully acknowledge scientific and technical assistance provided by Carme Carrion Ribas, Alyson Langley, Sarah A. Tabozzi and Jose Serrano as well as all partners in the Pegaso Fit for Future Study. We would also like to thank all the participating schools: students, parents, teachers and school principals for their commitment. Participating schools:
Bluecoat Beechdale Academy, Djanogly City Academy, Bluecoat Beechdale Academy, Armadale Academy, Lasswade High School, Liceo Scientifico Elio Vittorini, ITAS Giulio Natta, Escola Nou Patufet, IES Ramon de la Torre, Escola Fuster, Col.legi Manent Rambla, FEDAC Santa Coloma and Virgen del

Carmen.

PEGASO Fit For Future Consortium (in alphabetical order):

AQuAs: Carme Carrion, Mireia Espallargues, Santiago Felipe Gomez, Elisa

Puigdomenech.

ASPCAT: Conxa Castell.

BTB: Till Becker.

COVUNI: Ian Dunwell, Kim Bul.

CNR: Fulvio Adorni, Martina Caramenti, Valentina Ciociola, Chiara Crespi, Claudio Lafortuna, Gianfranco Modoni, Federica Prinelli, Giovanna Rizzo, Marco Sacco, Aleksandra Sojic, Sarah Tabozzi, Nithiya Jesuthasan.

CSEM: Olivier Grossenbacher, Mathieu Lemay, Enric Muntané Calvo.

EURECAT: Felip Miralles, Silvia Orte, Marc Solà, Filip Velickovski.

FPOLIMI: Mauro Brivio, Maria Renata Guarneri.

HES-SO: Leonardo Angelini, Maurizio Caon, Stefano Carrino, Elena Mugellini. LGATE: Cesare Delaini, Dalia Morosini.

LISPA: Marco Decandia, Sara Facchinetti, Andrea Migliavacca, Silvana Mura.

NEOSPERIENCE: Luca Bianchi, Marco Mazzola, Sandro Repetti.

POLIMI: Giuseppe Andreoni, Alessandra Mazzola, Paolo Perego, Carlo Emilio Standoli.

ROPARDO: Ciprian Candea, Gabriela Candea.

SIGLA: Massimiliano Azzolini, Luca Bianconi, Marco Costacurta, Cristiana Degano, Fabio Podda.

IMA: Antonio Ascolese, Lucia Pannese.

UEDIN: Lucy McCloughan, Janet Hanley, Yvonne Laird, Anne Martin, Brian

McKinstry, Rajeeb Rashid, George Scott.

UdL: Jose Serrano.

UNOTT: Sarah Atkinson, Sue Cobb, Laura Condon, Neil Coulson, Alexandra

Lang, Alyson Langley.

\section{Authors' contributions}

$A L, A M, B M, C C A, C L, E P, F A, F P, J H, L C, L M, M C, M E, R R ; S A, S F G, V C$ conceived the study protocol and designed the study interventions to work within schools in each of the separate sites involved. All listed authors contributed to the design and development of study materials, produced documents and contributed evidence for submission and review by appropriate ethics committees and engaged in recruitment activities to enlist schools and students for the trial of the technology. The multidisciplinary nature of this study required the involvement and expertise of academics and practitioners in health psychology, human factors and human computer interaction and clinicians working in child and adolescent health. The list of authors cover this range of disciplines and the geographical sites of the study, enabling the development of a protocol which integrates methods from multiple disciplines. The contributions of all personnel listed enabled the integrated, cross cultural work undertaken in this study. AM, EP, SFG wrote the first versions of the manuscript. All other authors contributed to the iterative writing and review of the manuscript. All authors read and approved the final manuscript.

\section{Funding}

This project was funded by research grants of the European Commission under the 7th Framework Programme (Call identifier: FP7-ICT-2013-10; Project number: 610727) and of the Instituto de Salud Carlos III through the project RD16/0001/ 0010 (Co-funded by European Regional Development Fund/European Social Fund "A way to make Europe"/"Investing in your future"). Funding has allowed the evaluation work package of the project to design the study, collect and analyse data and in writing the manuscript. AM was supported by the UK Medical Research Council (grant number MC_UU_12017/ 14) and the Scottish Government Chief Scientist Office (grant number SPHSU14).

\section{Availability of data and materials Not applicable.}

\section{Ethics approval and consent to participate}

Ethical approval has been granted by the clinical research ethics committee (CEIC) of all four intervention sites: South East Scotland Research Ethics Committee in Scotland and England (16/SS/0163; AMO SA1), Istituto di Ricovero e Cura a Carattere Scientifico (IRCCS) Policlinico of Milan in Italy (212_2016) and CEIC of Institut d'Investigació en Atenció Primaria de Salut 
Jordi Gol (IDIAP Jordi Gol) in Spain (P16/113). Participants and their guardians provided written informed consent.

\section{Consent for publication}

Not applicable.

\section{Competing interests}

The authors declare that they have no competing interests.

\section{Author details}

${ }^{1}$ Agency for Health Quality and Assessment of Catalonia (AQuAS), Catalan Department of Health, Roc Boronat 81-95, 2nd floor, 08005 Barcelona, Spain. ${ }^{2}$ Health Services Research on Chronic Patients Network (REDISSEC), Instituto de Salud Carlos III, Roc Boronat 81-95, 2nd floor, 08005 Barcelona, Spain. ${ }^{3} \mathrm{MRC} / \mathrm{CSO}$ Social and Public Health Sciences Unit, University of Glasgow, 200 Renfield Street, G2 3AX Glasgow, UK. ${ }^{4}$ Human Factors Research Group, Faculty of Engineering, University of Nottingham, University Park, NG7 2RD Nottingham, UK. ${ }^{5}$ National Research Council, Institute of Biomedical Technologies, Via Fratelli Cervi, 93, 20090 Segrate (MI), Italy. ${ }^{6}$ Programs Department Gasol Foundation, 26-28 Jaume I street, 08830 Sant Boi de Llobregat, Spain. ${ }^{7}$ GREpS. Health Education Research Group,Nursing and Phisiotherapy Department, University of Lleida, 2 Montserrat Roig street, 25198 Lleida, Spain. ${ }^{8}$ Usher Institute, University of Edinburgh, University of Edinburgh, NINE Edinburgh BioQuarter, 9 Little France Road, EH16 4UX Edinburgh, UK. ${ }^{9}$ National Research Council, Institute of Biomedical Technologies, Via Fratelli Cervi, 93, 20090 Segrate (MI), Italy. ${ }^{10}$ PRISM Research Group, Division of Primary Care, School of Medicine, University of Nottingham, Room 1404, Tower Building, University Park, NG7 2RD Nottingham, UK. ${ }^{11}$ Deanery of Clinical Sciences, College of Medicine and Veterinary Medicine, The Queen's Medical Research Institute, University of Edinburgh, Edinburgh BioQuarter, 47 Little France Crescent, EH16 4TJ Edinburgh, UK. ${ }^{12}$ College of Engineering \& School of Management, University of Applied Sciences and Arts Western Switzerland (HES-SO), chemin du musée 4n, 1700 Fribourg, Switzerland. ${ }^{13}$ Human Factors Research Group, Faculty of Engineering, The University of Nottingham, University Park, NG7 2RD Nottingham, UK. ${ }^{14}$ Consiglio Nazionale delle Ricerche, Istituto di Fisiologia Clinica, Piazza Ospedale Maggiore, 3, 20162 Milano, Italy. ${ }^{15}$ National Research Council, Institute of Biomedical Technologies, Via Fratelli Cervi, 93, 20090 Segrate (MI), Italy. ${ }^{16}$ School of Health and Social Care, Edinburgh Napier University, Sighthill Court, EH41 3ND Edinburgh, UK. ${ }^{17}$ Usher Institute, University of Edinburgh, NINE Edinburgh BioQuarter, 9 Little France Road, EH16 4UX Edinburgh, UK. ${ }^{18}$ Catalonia Public Health Agency, Catalan Department of Health, Roc Boronat 81-95, 3rd floor, 08005 Barcelona, Spain.

\section{Received: 19 October 2018 Accepted: 4 November 2019}

\section{Published online: 17 December 2019}

\section{References}

1. Viner R, Macfarlane A. Health promotion. BMJ. 2005;330(7490):527-9

2. Moreno LA, Gottrand F, Huybrechts I, Ruiz JR, González-Gross M, De Henauw S, HELENA Study Group. Nutrition and lifestyle in european adolescents: the HELENA (Healthy Lifestyle in Europe by Nutrition in Adolescence) study. Adv Nutr. 2014;5(5):615S-23S.

3. Sawyer SM, Afifi RA, Bearinger LH, Blakemore S-J, Dick B, Ezeh AC, et al. Adolescence: a foundation for future health. Lancet. 2012;379:1630-40

4. Black MM, Hurley KM. Investment in early childhood development. Lancet. 2014:384:1244-5

5. Dick B, Ferguson BJ. Health for the world's adolescents: a second chance in the second decade. J Adolesc Health. 2015;56:3-6.

6. World Health Organization (WHO): Adolescent development. 2015. http:// www.who.int/maternal_child_adolescent/topics/adolescence/development. Accessed 20 Nov 2017.

7. Ambrosini G. Childhood dietary patterns and later obesity: a review of the evidence. Proc Nutr Soc. 2014;73:137-46.

8. Rauner A, Mess F, Woll A. The relationship between physical activity, physical fitness and overweight in adolescents: a systematic review of studies published in or after 2000. BMC Pediatr. 2013;13:19.

9. Fatima Y, Doi SA, Mamun AA. Longitudinal impact of sleep on overweight and obesity in children and adolescents: a systematic review and biasadjusted meta-analysis. Obes Rev. 2015;16(2):137-49.

10. Liao Y, Liao J, Durand CP, Dunton GF. Which type of sedentary behaviour intervention is more effective at reducing body mass index in children? A meta-analytic review. Obes Rev. 2014;15(3):159-68.
11. Biddle SJ, Petrolini I, Pearson N. Nterventions designed to reduce sedentary behaviours in young people: a review of reviews. Br J Sports Med. 2014; 48(3):182-6.

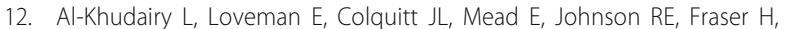
et al. Diet, physical activity and behavioural interventions for the treatment of overweight or obese adolescents aged 12 to 17 years. Cochrane Database Syst Rev. 2017;6:CD012691. https://doi.org/10.1002/ 14651858.CD012691.

13. Diethelm K, Jankovic N, Moreno LA, Huybrechts I, De Henauw S, De Vriendt $T$, et al. Food intake of European adolescents in the light of different foodbased dietary guidelines: results of the HELENA (Healthy Lifestyle in Europe by Nutrition in Adolescence) Study. Public Health Nutr. 2012. https://doi. org/10.1017/S1368980011001935.

14. Gallus S, Lugo A, Murisic B, Bosetti C, Boffetta P, La Vecchia C. Overweight and obesity in 16 European countries. Eur J Nutr. 2015. https://doi.org/10. 1007/s00394-014-0746-4.

15. Biro FM, Wien M. Childhood obesity and adult morbidities. Am J Clin Nutr. 2010;91(Suppl):1499S-505S.

16. NCD Risk Factor Collaboration (NCD-RisC). Worldwide trends in body-mass index, underweight, overweight, and obesity from 1975 to 2016: a pooled analysis of 2416 population-based measurement studies in 128.9 million children, adolescents, and adults. Lancet. 2017. https://doi.org/10.1016/ S0140-6736(17)32129-3.

17. Inchley J, Currie D, Young T, Samdal O, Torsheim T, Augustson L, et al. Growing up unequal: gender and socioeconomic differences in young people's health and well-being. Health behaviour in school-aged children (HBSC) study: international report from the 2013/2014 survey. WHO Regional Office for Europe. 2016. http://www.euro.who.int/_data/assets/ pdf_file/0003/303438/HSBC-No.7-Growing-up-unequal-Full-Report.pdf. Accessed 20 Nov 2017.

18. Oreskovic NM, Huang TT, Moon J. Integrating mHealth and Systems Science: A Combination Approach to Prevent and Treat Chronic Health Conditions. JMIR Mhealth Uhealth. 2015. https://doi.org/10.2196/mhealth.4150.

19. Matthew-Maich $N$, Harris L, Ploeg J, Markle-Reid M, Valaitis R, Ibrahim $S$, et al. Designing, Implementing, and Evaluating Mobile Health Technologies for Managing Chronic Conditions in Older Adults: A Scoping Review. JMIR Mhealth Uhealth. 2016. https://doi.org/10.2196/mhealth.5127.

20. Pinnock H, Hanley J, McCloughan L, Todd A, Krishan A, Lewis S, Stoddart A, Van der Pol M, MacNee W, Sheikh A, Pagliari C, McKinstry B. Effectiveness of telemonitoring integrated into existing clinical services on hospital admission for exacerbation of chronic obstructive pulmonary disease: researcher blind, multicentre, randomised controlled trial. BMJ. 2013. https:// doi.org/10.1136/bmj.f6070.

21. McKinstry B, Hanley J, Wild S, Pagliari C, Paterson M, Lewis S, Sheikh A, Krishan A, Stoddart A, Padfield P. Telemonitoring based service redesign for the management of uncontrolled hypertension: multicentre randomised controlled trial. BMJ. 2013, 2013. https://doi.org/10.1136/bmj.f3030.

22. Ferrer-Roca O, Gonzalez-Méndez D. Health 4.0 in the i2i era. Int J Reliable Quality E-Healthcare. 2012;1(1):43-57.

23. European Union. Eurostats. Being young in Europe today 2015. http://ec. europa.eu/eurostat/documents/3217494/6776245/KS-05-14-031-EN-N.pdf/1 8bee6f0-c181-457d-ba82-d77b314456b9. Accessed 20 Nov 2017.

24. Ng K, Tynjälä J, Kokko S. Ownership and Use of Commercial Physical Activity Trackers Among Finnish Adolescents: Cross-Sectional Study. JMIR Mhealth Uhealth. 2017. https://doi.org/10.2196/mhealth.6940.

25. Wong CA, Merchant RM, Moreno MA. Using social media to engage adolescents and young adults with their health. Healthc (Amst). 2014;2(4):220-4.

26. Vandewater EA, Denis LM. Media, social networking, and pediatric obesity. Pediatr Clin N Am. 2011;58(6):1509-19.

27. Turner T, Spruijt-Metz D, Wen CK, Hingle MD. Prevention and treatment of pediatric obesity using mobile and wireless technologies: a systematic review. Pediatric obesity. 2015. https://doi.org/10.1111/ijpo.12002.

28. Quelly SB, Norris AE, DiPietro JL. Impact of mobile apps to combat obesity in children and adolescents: A systematic literature review. J Spec Pediatr Nurs. 2016. https://doi.org/10.1111/jspn.12134.

29. Dute DJ, Bemelmans WJE, Breda J. Using Mobile Apps to Promote a Healthy Lifestyle Among Adolescents and Students: A Review of the Theoretical Basis and Lessons Learned. JMIR mHealth and uHealth. 2016:4(2):e39.

30. Michie S, van Stralen MM, West R. The behaviour change wheel: a new method for characterising and designing behaviour change interventions. Implement Sci. 2011;6(1):1. 
31. Standoli CE, Guarneri MR, Perego P, Mazzola M, Mazzola A, Andreoni G. A Smart Wearable Sensor System for Counter-Fighting Overweight in Teenagers. Sensors (Basel). 2016. https://doi.org/10.3390/s16081220.

32. Harrell JS, McMurray RG, Baggett CD, Pennell ML, Pearce PF, Bangdiwala SI. Energy costs of physical activities in children and adolescents. Med Sci Sports Exerc. 2005;37:329-36.

33. Personal Data and on the Free Movement of such Data," Official Journal of the EC, vol. 23, 1995. http://ec.europa.eu/justice/policies/privacy/docs/95-46ce/dir1995-46_part1_en.pdf. Accessed 20 Nov 2017.

34. European Union. Directive (EU) 2016/680. 2017. http://eur-lex.europa.eu/legalcontent/EN/TXT/?uri=uriserv: OJ.L_.2016.119.01.0089.01.ENG. Accessed 20 Nov 2017.

35. Serra-Majem L, Ribas L, Ngo J, Ortega RM, García A, Pérez-Rodrigo C, et al. Food, youth and the Mediterranean diet in Spain. Development of KIDMED, Mediterranean diet quality index in children and adolescents. Public Health Nutr. 2004;7(7):931-5.

36. Janz KF, Lutuchy EM, Wenthe P, Levy SM. Measuring activity in children and adolescents using self-report: PAQ-C and PAQ-A. Med Sci Sports Exerc. 2008; 40:767-72.

37. Rey-López JP, Ruiz JR, Ortega FB, Verloigne M, Vicente-Rodriguez G, GraciaMarco L, et al. Reliability and validity of a screen time-based sedentary behaviour questionnaire for adolescents: the HELENA study. Eur J Pub Health. 2012. https://doi.org/10.1093/eurpub/ckr040. Epub 2011 Apr 15.

38. Wolfson AR, Carskadon MA, Acebo C, Seifer R, Fallone G, Labyak SE, et al. Evidence for the validity of a sleep habits survey for adolescents. Sleep. 2003;26(2):213-6.

39. Turconi G, Celsa M, Rezzani C, Biino G, Sartirana MA, Roggi C. Reliability of dietary questionnaire on food habits, eating behaviours and nutritional knowledge of adolescents. Eur J Clin Nutr. 2003;57:753-63.

40. Deci EL, Ryan RM. Intrinsic Motivation and Self-Determination in Human Behavior, vol. 2. New York: Plenum; 1985. Williams GC, Deci EL: Internalization of biopsychosocial values by medical students: a test of selfdetermination theory J Pers Soc Psychol 70:767-779, 199

41. International Organization for Standardization (ISO). 2010: https://www.iso. org/standard/52075.html. Accessed 20 Nov 2017.

42. Usability.gov. 2017. https://www.usability.gov/how-to-and-tools/methods/ system-usability-scale.html. Accessed 20 Nov 2017.

43. Lewis JR, Mayes DK. Development and psychometric evaluation of the emotional metric outcomes (EMO) questionnaire. J Int J Hum Comput Int. 2014;30(9):685-702.

44. Gardner B, de Bruijn GJ, Lally P. A systematic review and meta-analysis of applications of the Self-Report Habit Index to nutrition and physical activity behaviours. Ann Behav Med. 2011. https://doi.org/10.1007/s12160-011-9282-0.

45. Michie S, Atkins L, West R. The behaviour change wheel: a guide to designing interventions. 1st ed. Silverback Publishing: London; 2014

46. Institute for Clinical Systems Improvement (ICSI). 2013. Guidelines on Prevention and management of obesity for children and adolescents. https://www.icsi.org/guidelines_more/catalog_guidelines_and_more/ catalog_guidelines/catalog_endocrine_guidelines/obesity_children/. Accessed 27 Nov 2017

47. Davis TL, DiClemente R, Prietula M. Taking mHealth Forward: Examining the Core Characteristics. JMIR Mhealth Uhealth. 2016. https://doi.org/10.2196/ mhealth.5659.

48. Shin JC, Kim J, Grigsby-Toussaint D. Mobile phone interventions for sleep disorders and sleep quality: systematic review. JMIR Mhealth Uhealth. 2017. https://doi.org/10.2196/mhealth.7244.

49. Flores Mateo G, Granado-Font E, Ferré-Grau C, Montaña-Carreras X. Mobile Phone Apps to Promote Weight Loss and Increase Physical Activity: A Systematic Review and Meta-Analysis. J Med Internet Res. 2015. https://doi. org/10.2196/jmir.4836

50. Schoeppe S, Alley S, Van Lippevelde W, Bray NA, Williams SL, Duncan MJ, et al. Efficacy of interventions that use apps to improve diet, physical activity and sedentary behaviour: a systematic review. Int J Behav Nutr Phys Act. 2016;13(1):127.

51. Badawy SM, Kuhns LM. Texting and Mobile phone app interventions for improving adherence to preventive behavior in adolescents: a systematic review. JMIR Mhealth Uhealth. 2017;5(4):e50. https://doi.org/10.2196/ mhealth.6837.
52. Lau PWC, Mphil LEY, Wong DP. PhD,2 RansdellL A Systematic Review of Information and Communication Technology-Based Interventions for Promoting Physical Activity Behavior Change in Children and Adolescents. J Med Internet Res. 2011;13(3):e48.

53. Network EQ, Enhancing the QUAlity and Transparency Of health Research (EQUATOR) Network. 2017. http://www.equator-network.org/. Accessed 20 Nov 2017.

\section{Publisher's Note}

Springer Nature remains neutral with regard to jurisdictional claims in published maps and institutional affiliations.
Ready to submit your research? Choose BMC and benefit from:

- fast, convenient online submission

- thorough peer review by experienced researchers in your field

- rapid publication on acceptance

- support for research data, including large and complex data types

- gold Open Access which fosters wider collaboration and increased citations

- maximum visibility for your research: over $100 \mathrm{M}$ website views per year

At BMC, research is always in progress.

Learn more biomedcentral.com/submissions 